\title{
¿NECESITAMOS EL TRATAMIENTO FARMACOLÓGICO EN EL TRASTORNO DE PERSONALIDAD? \\ DO WE NEED PHARMACOLOGICAL TREATMENT IN PERSONALITY DISORDER?
}

\author{
José Carlos Peláez ${ }^{a}$, Laura Reyes-Molón ${ }^{\text {b y }}$ Claire Teijeira-Levet ${ }^{c}$ \\ a Médico Psiquiatra. Hospital de Día de Trastornos de la Personalidad. \\ Hospital Clínico San Carlos.pelaez5@hotmail.com \\ b Médico Psiquiatra. Centros de Atención Integral a Drogodependientes (CAID Sur) de la Comunidad de Madrid. \\ c Médico Psiquiatra. Hospital Comarcal d'Inca. Palma de Mallorca. Illes Ballears.
}

Cómo referenciar este artículo/How to reference this article:

Peláez, J. C., Reyes-Molón, L. y Teijeira-Levet, C. (2013). ¿Necesitamos el tratamiento farmacológico en el trastorno de personalidad? [Do we need pharmacological treatment in personality disorder?] Acción Psicológica, 10(1), 97-108. http://dx.doi.org/10.5944/ap.10.1.7037

\section{Resumen}

Los trastornos de personalidad han cobrado una mayor importancia en la práctica clínica debido a las consecuencias socio-sanitarias que conllevan para los pacientes y a las dificultades de abordaje que suponen para los terapeutas. La falta de homogeneidad de las muestras y de eficacia de los tratamientos en los estudios de investigación ha generado controversias con respecto a su clasificación y su abordaje terapéutico. Un enfoque dimensional centrado en los rasgos de personalidad puede ser la clave para plantear una estrategia terapéutica exitosa. Dicha estrategia ha de incluir un tratamiento psicoterapéutico y, en los casos que lo precisen, farmacológico; siendo ambos individualizados y acordados con los pacientes. En este artículo nos centraremos en la eficacia de los tratamientos farmacológicos que más evidencia clínica han presentado hasta el momento.
Palabras clave: Trastorno de personalidad; tratamiento, psicofarmacología; técnicas psicológicas, sintomatología afectiva; comportamiento impulsivo.

\section{Abstract}

Personality disorders have become more important in clinical practice due to social and health consequences to the patients and the difficulties of approach found for therapists. The lack of homogeneity of the samples and effectiveness of treatments in research studies has generated controversy regarding their classification and therapeutic approach. A dimensional approach focused on personality traits may be the key to raising a successful therapeutic strategy. This strategy must include a psychotherapeutic and, in cases that require it, a pharmacological treatment, being both individualized and agreed with patients. In this article we will focus on the effectiveness of phar- 
macological treatments that more clinical evidence has supported them so far.

Keywords: personality Disorders; treatment; psychopharmacology; psychological techniques; affective symptoms; impulsive behavior.

\section{Introducción}

La psiquiatría, al igual que el resto de las especialidades médicas, ha evolucionado a lo largo del siglo pasado, pero con salvedades. Ha sido una evolución cuantitativa y cualitativamente diferente. Muchos de los avances realizados en otras ramas de la medicina han venido precedidos por el descubrimiento de la causa de las diferentes enfermedades, sin embargo en psiquiatría no se ha podido descubrir a ciencia cierta la causa de ningún trastorno. Por un lado la complejidad no solo estructural sino funcional del cerebro y por otro la necesidad de un mayor desarrollo tecnológico, han hecho que el actual conocimiento de cómo funciona nuestro cerebro sea todavía insuficiente para explicar las enfermedades mentales.

Pese a ello, se han ido descubriendo tratamientos que mejoraban los diversos trastornos mentales, más aun durante las últimas décadas. Al igual que en los principios de la medicina, la observación del efecto que diferentes sustancias tenían en el cuerpo hacia que esta se introdujeran como tratamiento; valgan como ejemplo la quinina o la digitalis. Algo similar ha sucedido en la psiquiatría desde el descubrimiento las propiedades antimaníacas de las sales de litio en 1949 (López Muñoz y Alamo González, 1998). El descubrimiento de diferentes fármacos ha precedido a la búsqueda de un correlato biológico en el cerebro que pudiera explicar la génesis de los trastornos mentales. El desarrollo por similitud de nuevas moléculas ha sido el motor del descubrimiento de nuevos fármacos y gracias a ello, en la actualidad, disponemos de una rica farmacopea para el tratamiento de los pacientes psiquiátricos.

La prevalencia del trastorno de personalidad (TP) se encuentra entre el 10-15\% de la población y puede llegar hasta el 50-60\% de los pacientes psiquiátricos ambulatorios (Diaz-
Marsa et al., 2008). Los trastornos de la personalidad suelen estar infradiagnosticados en la asistencia médica por lo que suelen pasar desapercibidos fuera de la asistencia psiquiátrica, sobretodo en aquellos con un alto grado de funcionabilidad (Sanislow y McGlashan, 1998). La comorbilidad existente con los TP tiene una contribución sobre el deterioro funcional que pueden presentar y produce una interferencia sobre el tratamiento que hay que proporcionar a este tipo de pacientes (Reich y Vasile, 1993).

La personalidad, patrón cualitativo de identidad que nos condiciona en la interacción con el entorno, puede ser parcialmente modificable. Pero además, se deben de tener en cuenta tanto los rasgos adaptativos como los desadaptativos para poder predecir la evolución de un paciente con un TP (Tyrer et al., 2007). A la par, una compensación de unos con otros puede llevar a no consultar nunca con un terapeuta pese a ciertas dificultades en algunas esferas. Con esto vemos que en algunos casos, ciertos rasgos de personalidad pueden variar en el tiempo, en la severidad o ser amortiguados por otros rasgos beneficiosos.

Otra línea de pensamiento nos podría llevar a la conclusión de que a través de la práctica clínica se han ido viendo los efectos beneficiosos de los fármacos en TP. Esta deducción sería rebatible tanto desde la evidencia de dichos tratamientos como desde la práctica clínica. Incluso un planteamiento teórico podría generar dudas sobre la conveniencia o no de un tratamiento farmacológico en los TP, dado que se pueden considerar alteraciones fuera de los trastornos psiquiátricos principales.

Tendríamos que pensar si según la noción de personalidad esta puede enfermar o no. Si profundizamos en el concepto de personalidad, esta se puede definir como la organización dinámica de los sistemas psico-biológicos que modulan la adaptación a la experiencia (Cloninger, 1987). Entonces, ¿podría responder una alteración de esta organización a un tratamiento farmacológico? Desde el abordaje que de la personalidad ha hecho la psicología se ha diferenciado entre temperamento y carácter para diferenciar una parte más biológica y otra más ambiental; por lo que esta distinción parece corresponder a una 
mayor diferenciación de los sistemas cerebrales (Silk, 1998). ¿Podría responder entonces una alteración en dichos sistemas cerebrales a un tratamiento farmacológico?

\section{¿Abordaje categorial o dimensional de los TP?}

Aunque la personalidad comienza a tenerse en cuenta desde tiempos de Hipócrates y Galeno, es en el siglo XX cuando comienza a abordarse en profundidad. En 1921, Allport abordó la personalidad describiendo diversos rasgos de personalidad (RP) y haciendo posteriormente una primera clasificación en rasgos cardinales, centrales y secundarios (Cervone y Pervin, 2008). En esa misma década Schneider realiza una distinción de 10 tipos de personalidades psicopáticas, siendo Jaspers, quien posteriormente realiza una discriminación entre el desarrollo de la personalidad y los procesos de la enfermedad (Livesley, 2001). Es en el DSM III cuando se marcan las diferencias entre los trastornos mentales (eje I) y los trastornos de personalidad (eje II), además de definir hasta 11 categorías de TP (American Psychiatric Association (Washington D.C.), 1983). Esta clasificación categorial hace que diversos criterios formen un trastorno, simplificando la complejidad de la personalidad.

Frente a este abordaje se desarrolla un abordaje dimensional, que inicia Eysenck con la diferenciación del neuroticismo o inestabilidad emocional, la extroversión y el psicoticismo (S. B. Eysenck, White y H. J. Eysenck, 1976). Se empiezan a definir así los rasgos de personalidad y se correlacionan estos con un sustrato biológico. El abordaje dimensional cobra una mayor importancia con diferentes autores como Gray, Zuckerman-Kuhlman, Millon o Costa y McCrae; llegando a aparecer diferentes dimensiones de la personalidad correlacionadas con una base psicobiológica (tabla 1). Cloninger introduce los conceptos de búsqueda de novedad, evitación del daño y dependencia de la recompensa como dimensiones de la personalidad evaluable y que se pueden correlacionar con una base neurobiológica (Cloninger, Svrakic y Przybeck, 1993).
Tabla 1

Dimensión de la Personalidad

y Neurotransmisor asociado

\begin{tabular}{ll}
\hline Neurotransmisor & Dimensión de la Personalidad \\
\hline Dopamina & Cognitivo/Perceptiva \\
Serotonina & Impulsividad/Agresión \\
Noradrenalina & Regulación afectiva \\
Adrenalina & Modulación de la Ansiedad \\
\hline
\end{tabular}

Nota. Adaptado de Siever y Davis (1991)

La concepción dimensional se basa en una serie de dominios con factores diferentes que los definen y que serviría para definir la personalidad tanto la normal como la patológica. Existe varios abordajes dimensionales entre los que se destacan los de Eysenck, McCrae y Costa o el de Cattell. Uno de los más estudiados ha sido el modelo de los Cinco Factores de McCrae y Costa que diferencia cinco dimensiones independientes de la personalidad: Neuroticismo, Extraversión, Apertura a la Experiencia, Amabilidad y Sentido de Responsabilidad. Este modelo ha sido validado en los en adultos y adolescentes y en estudios longitudinales para ver las modificaciones y comprobar la estabilidad del modelo (De Fruyt, De Bolle, McCrae, Terracciano y Costa, 2009; McCrae et al., 1999). Por ello ha servido de base en múltiples estudios de la personalidad. Estudiar la correlación genética existente con estos factores ha sido objetivo de revisión por metanálisis, se aúna así estudios genéticos y rasgos de personalidad (de Moor et al., 2012, Munafo, 2003).

Es difícil saber si tiene sentido tratar farmacológicamente o no a los trastornos TP pero no solo eso, sino que además surgen diversas cuestiones teóricas entorno a los conceptos de TP y de RP. Aunque en el DSM III se diferencia entre trastorno mental y TP, esta diferenciación se va haciendo cada vez más difusa. Se ha llegado a considerar a los TP como una parte previa dentro de un continuum de los trastornos mentales (Siever y Davis, 1991) y se llega a afirmar que no se podía saber si los trastornos de personalidad eran o no enfermedades mentales (Kendell, 2002). Para aumentar nuestra confusión a medida que se van realizando más estudios en 
cuanto a la personalidad se comienza a valorar la posibilidad de que los RP no permanezcan inamovibles a lo largo del tiempo (Tyrer et al., 2005), además se considera que, dentro de una disfuncionalidad, cada rasgo no tiene el mismo grado de severidad (Tyrer et al., 2007).

Tenemos por lo tanto dos abordajes para realizar una aproximación a los TP, uno dimensional y otro categorial. Ambos son conceptualmente muy diferentes pero si atendemos al diagnóstico categorial, es decir por los diferentes clases de TP, encontramos ciertas dificultades. Este tipo de diagnóstico presenta una fiabilidad baja, incluso si se mide en un mismo paciente y por un mismo evaluador, utilizando diferentes instrumentos psicométricos y evaluándolo en diferentes momentos (Ranger, Methuen, Rutter, Rao y Tyrer, 2004). Además existe una falta de acuerdo en el diagnóstico según los diversos TP específicos, esto es porque la mayoría de los criterios diagnósticos se solapan (Tyrer et al., 2007). Hasta un 29\% de los pacientes diagnosticados de trastorno esquizotípico cumplían criterios de trastorno límite de la personalidad (TLP) (Bender et al., 2001). También dentro de un abordaje categorial se llegan a encontrar un alto grado de comorbilidad entre el Cluster B (tabla 2) siendo el diagnóstico dimensional más apropiado que el categorial para establecer un diagnóstico en los TP (Westen, Shedler y Bradley, 2006).

Tabla 2

Comorbilidad en el DSM-IV utilizando un diagnóstico categorial

\begin{tabular}{lcccc}
\hline \multicolumn{1}{c}{ Tipo de TP } & TP Antisocial & TP Límite & TP Histriónico & TP Narcisista \\
\hline TP Antisocial & - & $30.7 \%$ & $50.0 \%$ & $40.7 \%$ \\
TP Límite & $66.0 \%$ & - & $59.0 \%$ & $50.8 \%$ \\
TP Histriónico & $44.7 \%$ & $28.7 \%$ & - & $40.7 \%$ \\
TP Narcisista & $51.1 \%$ & $29.7 \%$ & $57.1 \%$ & - \\
\hline
\end{tabular}

Nota. Adaptada de Westen et al. (2006)

Este problema, deducible de la práctica clínica, está siendo abordado en el borrador del DSM V (www.dsm5.org). Se propone un hibrido categorial-dimensional con 6 TP (Esquizotipico, Antisocial, Límite, Narcisista, Evitativo y Obsesivo-compulsivo) y 5 dominios en los rasgos de personalidad (afectividad negativa, indiferencia, antagonismo, desinhibición vs compulsión y psicoticismo).

La redefinición del concepto de TP y la combinación de un modelo categorial con otro dimensional en la línea de Costa y McCrae (1992) y Oldham y Skodol (2000) son algunos de los cambios importantes que en los TP podría introducir el DSM V. Asimismo, se redefine los TP centrándose en los fallos de adaptación, dando pie a incluir una valoración dimensional (Esbec y Echeburua, 2011). Poder tener un correlato dimensional con uno biológico y así poder diferenciar la parte genética de la parte ambiental en los rasgos de persona- lidad ha sido objeto de estudio en las últimas décadas (Bouchard y McGue, 2003).

Hemos de volver al diagnóstico dimensional como un modo para poder clasificar la personalidad de los individuos. De tal manera que si se diagnosticara según los cluster del DSM IV-TR (American Psychiatric Association y American Psychiatric Association. Task Force on DSM-IV, 2000) o teniendo en cuenta un abordaje dimensional (como posiblemente ocurra en el DSM V) se ganaría en precisión y así se podrían unificar criterios de abordaje y tratamiento.

\section{¿Tratamiento solamente farmacológico?}

Antes de plantearse un tratamiento farmacológico hay que tener en cuenta una serie de factores que no se pueden pasar por alto: un correcto diagnóstico, un estudio de los rasgos de 
personalidad, integrar el tratamiento farmacológico dentro de un plan terapéutico que debe incluir un abordaje psicoterapéutico y familiar, negociación de los objetivos a corto y largo plazo, ideación de un plan de actuación en caso de crisis y elaborar una continuidad de cuidados orientada a la rehabilitación u obtención del mayor grado de funcionabilidad posible en los casos más graves. En cuanto al tratamiento farmacológico cabría considerar la indicación y duración de los mismos a fin de evitar, en la medida de lo posible, la polifarmacia y los tratamientos de duración indeterminada y dar siempre la mayor información sobre el tratamiento. Dado que no existe ningún tratamiento indicado en los TP hay que prever alternativas farmacológicas evitando el paternalismo y teniendo en cuenta la autonomía del paciente.

El malestar que padece el paciente y las alteraciones percibidas por el psiquiatra pueden estar detrás de los primeros abordajes psicofarmacológicos. Posteriormente tras el desarrollo de un abordaje dimensional se han ido delimitando más las dianas terapéuticas. Así nos encontrarnos que de manera específica, dentro del TLP, la agresividad, la impulsividad, la ansiedad, los síntomas pseudo-psicóticos y la inestabilidad afectiva, han sido las dimensiones más estudiadas (American Psychiatric
Association, 2001). Sin embargo son la regulación afectiva, los patrones de acción/impulsividad, la organización cognitiva y la ansiedad/inhibición los ejes biológicos de la personalidad que probablemente tienen un correlato psicobiológico generalizable al resto de TP (Carrasco, 1997; Skodol et al., 2002).

$\mathrm{Al}$ ser los TP tan diversos y la demanda de recursos tan diferente dependiendo del tipo, es muy difícil hacer propuestas extensibles al resto de TP. Bender (2001), realiza un estudio sobre la utilización de recursos por parte de los TP (Tabla 3) (Bender et al., 2001). Los TP utilizan los recursos psicoterapéuticos en gran medida: cualquier tipo de psicoterapia $96 \%$; tratamiento hospitalario (hospitalización de agudos u hospital de día) 48\%; tratamiento farmacológico $81 \%$. Pero si nos fijamos en los porcentajes por los TP del Cluster A y B se diferencian del tipo evitativo y obsesivo compulsivo al ser los que más recursos utilizan. Dentro de todos los TP, la mayor utilización de recursos se realiza por los pacientes con TLP. Debido a ello han sido el objetivo de la mayor parte de los estudios y se han elaborado guías de tratamiento para un correcto abordaje [Lalucat Jo, Casañas Sánchez, Estrada Sabadell, Mas Expósito y Teixidó Casas, 2011; National Collaborating Centre for Mental Health (UK), 2009].

\section{Tabla 3}

Utilización de recursos en los Trastornos de Personalidad

\begin{tabular}{|c|c|c|c|c|c|}
\hline $\begin{array}{c}\text { Tipo } \\
\text { de Tratamiento }\end{array}$ & $\begin{array}{c}\text { Trastornos } \\
\text { de Personalidad }\end{array}$ & $\begin{array}{c}\text { Trastorno } \\
\text { Esquizotípico }\end{array}$ & $\begin{array}{l}\text { Trastorno } \\
\text { Límite }\end{array}$ & $\begin{array}{l}\text { Trastorno } \\
\text { Evitativo }\end{array}$ & $\begin{array}{c}\text { Trastorno } \\
\text { Obsesivo-Compulsivo }\end{array}$ \\
\hline $\begin{array}{l}\text { Psicoterapia } \\
\text { individual }\end{array}$ & $93 \%$ & $92 \%$ & $95 \%$ & $93 \%$ & $94 \%$ \\
\hline Psicoterapia grupal & $39 \%$ & $41 \%$ & $56 \%$ & $32 \%$ & $26 \%$ \\
\hline $\begin{array}{l}\text { Terapia de familia } \\
\text { o de pareja }\end{array}$ & $34 \%$ & $26 \%$ & $42 \%$ & $26 \%$ & $36 \%$ \\
\hline $\begin{array}{l}\text { Grupo de } \\
\text { autoayuda }\end{array}$ & $31 \%$ & $32 \%$ & $41 \%$ & $22 \%$ & $27 \%$ \\
\hline Centro de día & $22 \%$ & $24 \%$ & $37 \%$ & $17 \%$ & $9 \%$ \\
\hline Hospital de día & $14 \%$ & $20 \%$ & $24 \%$ & $7 \%$ & $6 \%$ \\
\hline $\begin{array}{l}\text { Hospitalización } \\
\text { psiquiátrica }\end{array}$ & $46 \%$ & $20 \%$ & $72 \%$ & $36 \%$ & $23 \%$ \\
\hline
\end{tabular}

Nota. Adaptado de Bender et al. (2001) 
La polifarmacia puede ser un riesgo dentro de los trastornos de personalidad debido a la inespecificidad sintomatológica, la automedicación, las múltiples demandas, la falta de estabilidad o incluso el agotamiento de recursos psicoterapéuticos o de rehabilitación. La prescripción de tratamientos dentro de los TP podría seguir un curso similar al de la tabla 4, pero es probable que en nuestro medio, la uti- lización de tratamiento antipsicótico y ansiolíticos, tanto en TP del cluster A como del B, sea mayor que los encontrados en este estudio (Bender et al., 2001). Un estudio realizado en nuestro medio con TLP, encuentra un porcentaje de 2,7 medicamentos por paciente, donde un $56 \%$ estaba tomando $\geq 3$ medicamentos y un $30 \% \geq 4$ medicamentos y solo un $6 \%$ estaba sin tratamiento (Pascual et al., 2010).

Tabla 4

Distribución del tratamiento psicofarmacológico según el tipo de TP.

\begin{tabular}{lcccc}
\hline \multicolumn{1}{c}{ Tipo de medicación } & $\begin{array}{c}\text { Trastorno } \\
\text { Esquizotipico }\end{array}$ & Trastorno Límite & $\begin{array}{c}\text { Trastorno } \\
\text { Evitativo }\end{array}$ & $\begin{array}{c}\text { Trastorno } \\
\text { Obsesivo-Compulsivo }\end{array}$ \\
\hline Ansiolíticos & $22 \%$ & $35 \%$ & $20 \%$ & $14 \%$ \\
Hipnóticos & $4 \%$ & $6 \%$ & $2 \%$ & $3 \%$ \\
Estabilizadores del ánimo & $12 \%$ & $27 \%$ & $7 \%$ & $2 \%$ \\
Antipsicóticos & $10 \%$ & $10 \%$ & $5 \%$ & $<1 \%$ \\
Antidepresivos & $52 \%$ & $61 \%$ & $57 \%$ & $44 \%$ \\
\hline
\end{tabular}

Nota. Adaptada de Bender, 2001

La American Psychiatry Association (APA) (American Psychiatric Association, 2001) presento una guía clínica en el 2001 que sirvió para el tratamiento del TLP y que ha servido como ejemplo en la práctica clínica para el tratamiento del resto de TP; aunque las indicaciones que mencionaban han sido rebatidas variando así las indicaciones en la guía NICE para el abordaje del TLP (National Collaborating Centre for Mental Health (UK), 2009). En esa guía de la APA se realizaban las siguientes indicaciones según la sintomatología:

- Síntomas de desregulación afectiva (labilidad emocional, sensibilidad al rechazo, ira inapropiada o los estallidos de ira, «bajones» en el estado de ánimo): primero ISRS, si no es efectivo o tiene una eficacia parcial, cambiar de ISRS. Si continúa sin ser efectivo o lo es solo parcialmente, añadir antipsicóticos (si existe ira) o clonazepam (si existe ansiedad). Si todavía no existe una respuesta cambiar a IMAO y en última instancia si no existe respuesta cambiar a un estabi- lizador del ánimo (litio, carbamazepina o ácido valproico).

- Impulsividad o síntomas de descontrol conductual (agresión impulsiva, autoagresividad, conductas compulsivas perjudiciales sí mismo (por ejemplo, promiscuidad sexual, abuso de sustancias, el gasto imprudente, etc.): Se inicia un tratamiento con un ISRS y si no es efectivo o tiene una eficacia parcial se utilizarían bajas dosis de antipsicótico de primera generación. En caso de no ser efectiva ni una ni otra o la combinación de ambas se podría añadir un estabilizador del ánimo o un IMAO. En última instancia se recomienda el uso de antipsicóticos de segunda generación.

- Distorsiones cognitivas (el paciente es muy suspicaz, ideación autorreferencial, ideación paranoide, ilusiones, desrealización, despersonalización, o síntomas similares a la alucinaciones). Recomiendan utilizar dosis bajas de un antipsicótico (de primera o segunda generación) e incrementar la dosis si no existe una 
adecuada eficacia. En caso de no ser efectivo o serlo de forma parcial se recomienda añadir un ISRS o un IMAO, cambiar de antipsicótico o pasar a clozapina.

Pese a que no existen evidencias para el tratamiento sintomatológico de los pacientes con TP, diferentes estudios con diversos tipos de fármacos han puesto de manifiesto las evidencias existentes con respecto a los fármacos que se exponen en la tabla 5 (Ingenhoven, Lafay, Rinne, Passchier y Duivenvoorden; Lieb, Vollm, Rucker, Timmer y Stoffers; Nose, Cipriani, Biancosino, Grassi y Barbui, 2006; Rinne, van den Brink, Wouters y van Dyck, 2002; Tyrer y Bateman, 2004). Existen estudios que evidencian la mejoría en algunos as- pectos dimensionales como por ejemplo: la fluoxetina ha mostrado una eficacia en la falta de control, en la disminución de la impulsividad, en la auto-agresividad y en la sensación de rechazo (Coccaro, Harvey, KupsawLawrence, Herbert y Bernstein, 1991). Los inhibidores de la receptación de serotonina se han mostrado cambios en algunos rasgos de la personalidad en pacientes que los han tomado de forma crónica o sub-crónica, entres los cambios que se ha producido se han comprobado una disminución del neuroticismo y un incremento de la extraversión en pacientes con un trastorno depresivo y tratados con paroxetina (Tang et al., 2009) o solo una disminución del neuroticismo con la sertralina (Simmons y Allen, 2011).

\section{Tabla 5}

Tabla resumen sobre el tratamiento psicofarmacológico

\begin{tabular}{|c|c|c|}
\hline \multicolumn{2}{|c|}{ Tratamiento } & \multirow{2}{*}{$\begin{array}{l}\text { Síntomas } \\
\text { Reducción de: } \\
\text { - Ira inapropiada } \\
\text { - Impulsividad/agresión } \\
\text { - Problemas interpersonales }\end{array}$} \\
\hline $\begin{array}{l}\text { Antipsicóticos de primera } \\
\text { generación }\end{array}$ & Haloperidol & \\
\hline & Decanoato de flucopentixol & Reducción de las conductas autolíticas \\
\hline \multirow{3}{*}{$\begin{array}{l}\text { Antipsicóticos de segunda } \\
\text { generación }\end{array}$} & Aripiprazol & $\begin{array}{l}\text { Reducción de: } \\
\text { - Ira inapropiada } \\
\text { - Síntomas psicóticos } \\
\text { - Impulsividad } \\
\text { - Problemas interpersonales } \\
\text { - Impulsividad/agresión }\end{array}$ \\
\hline & Risperidona & $\begin{array}{l}\text { Reducción de: } \\
\text { - Impulsividad/agresión } \\
\text { - Problemas interpersonales } \\
\text { - Síntomas psicóticos }\end{array}$ \\
\hline & Olanzapina & $\begin{array}{l}\text { Reducción de: } \\
\text { - Inestabilidad afectiva } \\
\text { - Ira inapropiada } \\
\text { - Síntomas psicóticos } \\
\text { - Ansiedad } \\
\text { - Impulsividad/agresión } \\
\text { - Problemas interpersonales }\end{array}$ \\
\hline
\end{tabular}




\begin{tabular}{|c|c|c|}
\hline \multicolumn{2}{|c|}{ Tratamiento } & Síntomas \\
\hline \multirow{3}{*}{ Estabilizadores del ánimo } & Ácido Valproico & $\begin{array}{l}\text { Reducción de: } \\
\text { - Problemas interpersonales } \\
\text { - Síntomas depresivos } \\
\text { - Ira inapropiada (no concluyente) }\end{array}$ \\
\hline & Lamotrigina & $\begin{array}{l}\text { Reducción de: } \\
\text { - Impulsividad } \\
\text { - Ira inapropiada } \\
\text { - Problemas interpersonales }\end{array}$ \\
\hline & Topiramato & $\begin{array}{l}\text { Reducción de: } \\
\text { - Impulsividad } \\
\text { - Problemas interpersonales }\end{array}$ \\
\hline \multirow{3}{*}{ Antidepresivos } & Amitriptilina (ADT) & $\begin{array}{l}\text { Reducción de: } \\
\text { - Síntomas depresivos } \\
\text { - Ira inapropiada } \\
\text { - Disregulación afectiva }\end{array}$ \\
\hline & ISRSs & $\begin{array}{l}\text { Reducción de: } \\
\text { - Síntomas depresivos } \\
\text { - Ira inapropiada } \\
\text { - } \quad \text { Disregulación afectiva }\end{array}$ \\
\hline & Fenelcina (IMAO) & $\begin{array}{l}\text { Reducción de: } \\
\text { - Síntomas depresivos } \\
\text { - Ansiedad } \\
\text { - Patología psiquiátrica } \\
\text { Mejoría del estado de salud mental }\end{array}$ \\
\hline
\end{tabular}

Nota. ATD: antidepresivos tricíclicos; ISRSs: inhibidores de la recaptación de serotonina; IMAO: Inhibidor de la monoamino-oxidasa.

No existe la indicación para un tipo de síntoma dentro de los TP ni estudios que demuestren la eficacia de un fármaco sobre una dimensión específica. Sin embargo, desde un punto de vista farmacológico vemos que existen evidencias de que algunos tratamientos farmacológicos pueden ser efectivos en algunas alteraciones dimensionales, defendiendo un abordaje sintomatológico específico (Lieb et al., 2010), no así categorial. Es probable que en la práctica clínica diaria se usen más fármacos que los anteriormente expuestos aunque sea con los mismos objetivos.

\section{Conclusiones}

Existen dimensiones de la personalidad con un correlato biológico que son variables en el tiempo y en la intensidad (Tyrer y Bateman, 2004). Frente al abordaje dimensional existe uno categorial que presenta ciertas restricciones, como por ejemplo que los límites entre las categorías no son claros y además algunos criterios se pueden dar concomitantemente en muchas de las categorías diagnósticas (Soloff, 1998).

Las dimensiones están integradas por rasgos de la personalidad, existiendo una diferenciación cualitativa entre los rasgos funcionales y disfuncionales que varían el pronóstico de un mismo TP (Tyrer, Duggan y Coid, 2003). Un factor que no se pude obviar y que se ha de tener en cuenta es la diferencia que existe entre aquellos pacientes con un TP que buscan el tratamiento y aquellos que rechazan el mismo (Tyrer, Mitchard, Methuen, y Ranger, 2003). 
Con todo los TP tienen sintomatología que puede ser deteriorante y complicada produciendo en ocasiones una gran discapacidad, lo que les lleva a la utilización de gran cantidad de recursos en comparación con otros trastornos mentales (Bender et al., 2001).

El abordaje farmacológico en el TP podría cobrar sentido sobre todo en el tratamiento de alguna sintomatología, o incluso de manera excepcional algunos de los rasgos dentro de una dimensión (que sean graves o perturbadores) y en el tratamiento de una co-morbilidad psiquiátrica existente (abuso de sustancias, trastornos de la conducta alimentaria, trastorno ansioso-depresivo, etc.). Hay que tener en cuenta que los pacientes obtienen el máximo beneficio de un abordaje psicoterapéutico y farmacológico (Oldham, Bender, Skodol, Gabbard y American Psychiatric Publishing Inc, 2007), si bien existen estrategias farmacológicas más especificas para síntomas concretos dentro de los diferentes TP (Marín y Fernández, 2007). El abordaje farmacológico se ha centrado sobre todo en el TLP pero también en los síntomas individuales o conductas asociadas con un TP (por ejemplo, autolesiones repetidas, inestabilidad emocional marcada, elevada suspicacia, alteraciones perceptivas).

Los TP son susceptibles de tratamiento farmacológico en aquellos casos que por la gravedad o la intensidad del trastorno muestren que el abordaje psicoterapéutico se muestra insuficiente. Los casos más graves precisan de una gran cantidad de recursos hospitalarios y extra hospitalarios, llegando a ser muy frecuente la polifarmacia. Un plan de tratamiento individualizado evitará la iatrogenia o los tratamientos cronificados, dado que pueden presentar periodos de tiempo en los que se recrudece la sintomatología y periodos en los que esta sea menor.

No se puede obviar que los estudios existentes pueden tener una diferencia importante entre los pacientes que participan en los estudios y aquellos que acuden a consulta, pudiendo por ello existir diferentes recomendaciones de tratamiento. Una buena alianza terapéutica podría hacer que algunas intervenciones terapéuticas tengan mejores resultados, incluidos beneficios farmacológicos. Por todo ello se puede concluir que la individualización de un tratamiento psicoterapéutico que, cuando sea preciso, se complemente con un tratamiento farmacológico es el abordaje idóneo independientemente de la gravedad del caso.

\section{Referencias}

American Psychiatric Association. (2000). Diagnostic and statistical manual of mental disorders: DSM-IV-TR (4th ed.). Washington, DC, EE.UU.: Autor.

American Psychiatric Association (1983). Breviario DSM-III: criterios diagnósticos [DSM-III: diagnostic criteria]. Barcelona, España: Masson.

Bender, D. S., Dolan, R. T., Skodol, A. E., Sanislow, C. A., Dyck, I. R., McGlashan, T. H., Tracie Shea, M., Zanarini, M.C., Oldham, J.M., Gunderson, J.G. (2001). Treatment utilization by patients with personality disorders. American Journal of Psychiatry, 158(2), 295-302.

Bouchard, T. J., Jr. y McGue, M. (2003). Genetic and environmental influences on human psychological differences. Journal of Neurobiology, 54(1), 4-45.

Carrasco, J. L. (1997). Enfoque psicobiologico de los trastornos de personalidad [Psychobiological approach to personality disorders]. Actas LusoEspanolas de Neurologia, Psiquiatria y Ciencias Afines, 25(4), 207-216.

Cervone, D. y Pervin, L. A. (2008). Personality: theory and research (10. ${ }^{\mathrm{a}}$ Ed.). Hoboken, NJ: Wiley.

Cloninger, C. R. (1987). A systematic method for clinical description and classification of personality variants. A proposal. Archives of General Psychiatry, 44(6), 573-588.

Cloninger, C. R., Svrakic, D. M. y Przybeck, T. R. (1993). A psychobiological model of temperament and character. Archives of General Psychiatry, 50, 975-990.

Coccaro, E. F., Harvey, P. D., Kupsaw-Lawrence, E., Herbert, J. L. y Bernstein, D. P. (1991). Development of neuropharmacologically based behavioral assessments of impulsive aggressive behavior. Journal of Neuropsychiatry and Clinical Neurosciences, 3(2), S44-51. 
De Fruyt, F., De Bolle, M., McCrae, R. R., Terracciano, A. y Costa, P. T., Jr. (2009). Assessing the universal structure of personality in early adolescence: The NEO-PI-R and NEO-PI-3 in 24 cultures. Assessment, 16(3), 301-311.

De Moor, M. H., Costa, P. T., Terracciano, A., Krueger, R. F., de Geus, E. J., Toshiko, T.,... y Boomsma, D.I. (2012). Meta-analysis of genome-wide association studies for personality. Molecular Psychiatry, 17(3), 337-49.

Diaz-Marsa, M., Gonzalez Bardanca, S., Tajima, K., Garcia-Albea, J., Navas, M. y Carrasco, J. L. (2008). Psychopharmacological treatment in borderline personality disorder. Actas Españolas de Psiquiatría, 36(1), 39-49.

Esbec, E. y Echeburua, E. (2011). New criteria for personality disorders in DSM-V. Actas Españolas de Psiquiatría, 39(1), 1-11.

Eysenck, S. B., White, O. y Eysenck, H. J. (1976). Personality and mental illness. Psychological Reports, 39(3), 1011-1022.

Gabbard, G.O. (2001). Guideline for the treatment of patients with borderline personality disorder. Am J Psychiatry, 158:1-3.

Ingenhoven, T., Lafay, P., Rinne, T., Passchier, J. y Duivenvoorden, H. (2010). Effectiveness of pharmacotherapy for severe personality disorders: meta-analyses of randomized controlled trials. Journal of Clinical Psychiatry, 71(1), 14-25.

Kendell, R. E. (2002). The distinction between personality disorder and mental illness. British Journal of Psychiatry, 180, 110-115.

Lalucat Jo, L., Casañas Sánchez, R., Estrada Sabadell, M., Mas Expósito, L. y Teixidó Casas, M. (Eds.). (2011). Guía de práctica clínica sobre trastorno límite de la personalidad [Clinical practice guideline about borderline personality disorder]. Barcelona, España: Departamento de Salud de la Generalitat de Catalunya.

Lieb, K., Vollm, B., Rucker, G., Timmer, A. y Stoffers, J. M. (2010). Pharmacotherapy for borderline personality disorder: Cochrane systematic review of randomised trials. British Journal of Psychiatry, 196(1), 4-12.

Livesley, W. J. (2001). Handbook of personality disorders: theory, research, and treatment. New York: Guilford Press.
López Muñoz, F. y Alamo González, C. (1998). Historia de la neuropsicofarmacología: Una nueva aportación a la terapéutica farmacológica de los trastornos del Sistema Nervioso Central. [History of neuropsychopharmacology: A new contribution to pharmacological therapy of disorders of the Central Nervous System.]. Alcalá de Henares, España: Universidad de Alcalá.

Marín López, J.L., y Fernández Guerrero, M.J. (2007). Tratamiento farmacológico de los trastornos de personalidad [Pharmacological treatment of personality disorders]. Clinica y Salud, 18, 259-285.

McCrae, R. R., Costa, P. T., Jr., Pedroso de Lima, M., Simoes, A., Ostendorf, F., Angleitner, A.,... y Piedmont, R. L. (1999). Age differences in personality across the adult life span: parallels in five cultures. Developmental Psychology, 35, 466-477.

Munafo, M. R., Clark, T. G., Moore, L. R., Payne, E., Walton, R. y Flint, J. (2003). Genetic polymorphisms and personality in healthy adults: a systematic review and meta-analysis. Molecular Psychiatry, 8(5), 471-484.

National Collaborating Centre for Mental Health (UK). (2009). Borderline personality disorder : treatment and management. Leicester: British Psychological Society.

Nose, M., Cipriani, A., Biancosino, B., Grassi, L. y Barbui, C. (2006). Efficacy of pharmacotherapy against core traits of borderline personality disorder: meta-analysis of randomized controlled trials. International Clinical Psychopharmacology, 21(6), 345-353.

Oldham, J. M., Bender, D. S., Skodol, A. E., Gabbard, G. O. y American Psychiatric Publishing Inc. (2007). Tratado de los trastornos de la personalidad [Textbook of personality disorders]. Barcelona, España: Elservier Masson.

Pascual, J. C., Martin-Blanco, A., Soler, J., Ferrer, A., Tiana, T., Alvarez, E., Perez, V. (2010). A naturalistic study of changes in pharmacological prescription for borderline personality disorder in clinical practice: from APA to NICE guidelines. International Clinical Psychopharmacology, 25(6), 349-355.

Ranger, M., Methuen, C., Rutter, D., Rao, B. y Tyrer, P. (2004). Prevalence of personality disorder in the case-load of an inner-city assertive outreach team. Psychiatric Bulletin, 28(12), 441-443. 
Reich, J. H. y Vasile, R. G. (1993). Effect of personality disorders on the treatment outcome of axis I conditions: an update. Journal of Nervous and Mental Disease, 181(8), 475-484.

Rinne, T., van den Brink, W., Wouters, L. y van Dyck, R. (2002). SSRI treatment of borderline personality disorder: a randomized, placebocontrolled clinical trial for female patients with borderline personality disorder. American Journal of Psychiatry, 159(12), 2048-2054.

Sanislow, C. A. y McGlashan, T. H. (1998). Treatment outcome of personality disorders. Canadian Journal of Psychiatry, 43(3), 237-250.

Siever, L. J. y Davis, K. L. (1991). A psychobiological perspective on the personality disorders. American Journal of Psychiatry, 148(12), 1647-1658.

Silk, K. R. (1998). Biology of personality disorders. Washington, D.C.: American Psychiatric Press.

Simmons, J. G. y Allen, N. B. (2011). Mood and personality effects in healthy participants after chronic administration of sertraline. Journal of Affective Disorders, 134(1-3), 377-385.

Skodol, A. E., Siever, L. J., Livesley, W. J., Gunderson, J. G., Pfohl, B. y Widiger, T. A. (2002). The borderline diagnosis II: biology, genetics, and clinical course. Biological Psychiatry, 51, 951-963.

Soloff, P. H. (1998). Algorithms for pharmacological treatment of personality dimensions: symptomspecific treatments for cognitive-perceptual, affective, and impulsive-behavioral dysregulation. Bulletin of The Menninger Clinic, 62(2), 195-214.

Tang, T. Z., DeRubeis, R. J., Hollon, S. D., Amsterdam, J., Shelton, R. y Schalet, B. (2009). Personality change during depression treatment: a placebo-controlled trial. Archives of General Psychiatry, 66, 1322-1330.

Tyrer, P. y Bateman, A. W. (2004). Drug treatment for personality disorders. Advances in Psychiatric Treatment, 10(5), 389-398.

Tyrer, P., Coombs, N., Ibrahimi, F., Mathilakath, A., Bajaj, P., Ranger, M.,... y Din, R. (2007). Critical developments in the assessment of personality disorder. The British Journal of Psychiatry Suppl, 49, s51-59.

Tyrer, P., Cooper, S., Seivewright, H., Duggan, C., Rao, B. y Hogue, T. (2005). Temporal reliability of psychological assessments for patients in a special hospital with severe personality disorder: a preliminary note. Criminal Behaviour and Mental Health, 15(2), 87-92.

Tyrer, P., Duggan, C. y Coid, J. (2003). Ramifications of personality disorder in clinical practice. The British Journal of Psychiatry, 182(44), s1-s2.

Tyrer, P., Mitchard, S., Methuen, C. y Ranger, M. (2003). Treatment rejecting and treatment seeking personality disorders: Type R and Type S. Journal of Personality Disorders, 17, 263-268.

Westen, D., Shedler, J. y Bradley, R. (2006). A prototype approach to personality disorder diagnosis. American Journal of Psychiatry, 163, 846-856. 
